\title{
Effects of carnosine and hypothermia combination therapy on hypoxic-ischemic brain injury in neonatal rats
}

Jun Chul Byun, MD, PhD ${ }^{1, *}$, Seong Ryong Lee, MD, PhD², Chun Soo Kim, MD, PhD ${ }^{3}$

${ }^{1}$ Department of Pediatrics, Daegu Fatima Hospital, Daegu, Korea; ${ }^{2}$ Department of Pharmacology, Keimyung University School of Medicine, Daegu, Korea; ${ }^{3}$ Department of Pediatrics, Keimyung University School of Medicine, Daegu, Korea

Background: Carnosine has antioxidative and neuroprotective properties against hypoxic-ischemic (HI) brain injury. Hypothermia is used as a therapeutic tool for HI encephalopathy in newborn infants with perinatal asphyxia. However, the combined effects of these therapies are unknown.

Purpose: Here we investigated the effects of combined carnosine and hypothermia therapy on $\mathrm{HI}$ brain injury in neonatal rats.

Methods: Postnatal day 7 (P7) rats were subjected to HI brain injury and randomly assigned to 4 groups: vehicle; carnosine alone; vehicle and hypothermia; and carnosine and hypothermia. Carnosine $(250 \mathrm{mg} / \mathrm{kg})$ was intraperitoneally administered at 3 points: immediately following $\mathrm{HI}$ injury, 24 hours later, and 48 hours later. Hypothermia was performed by placing the rats in a chamber maintained at $27^{\circ} \mathrm{C}$ for 3 hours to induce whole-body cooling. Sham-treated rats were also included as a normal control. The rats were euthanized for experiments at P10, P14, and P35. Histological and morphological analyses, in situ zymography, terminal deoxynucleotidyl transferasemediated dUTP nick end-labeling (TUNEL) assays, and immunofluorescence studies were conducted to investigate the neuroprotective effects of the various interventional treatments. Results: Vehicle-treated P10 rats with HI injury showed an increased infarct volume compared to sham-treated rats during the triphenyltetrazolium chloride staining study. Hematoxylin and eosin staining revealed that vehicle-treated $\mathrm{P} 35$ rats with $\mathrm{HI}$ injury had decreased brain volume in the affected hemisphere. Compared to the vehicle group, carnosine and hypothermia alone did not result in any protective effects against $\mathrm{HI}$ brain injury. However, a combination of carnosine and hypothermia effectively reduced the extent of brain damage. The results of in situ zymography, TUNEL assays, and immunofluorescence studies showed that neuroprotective effects were achieved with combination therapy only.

Conclusion: Carnosine and hypothermia may have synergistic neuroprotective effects against brain damage following $\mathrm{HI}$ injury.
Keywords: Carnosine, Hypothermia, Hypoxia-ischemic brain injury

\section{Key message}

Question: How can we further improve the neuroprotective effects of hypothermia in newborns with hypoxic-ischemic (HI) brain injury?

Finding: Combination carnosine and hypothermia therapy effectively reduced brain damage in neonatal rats. The in situ zymography, terminal deoxynucleotidyl transferase-mediated dUTP nick end-labeling assay, and immunofluorescence study results showed that neuroprotective effects were achieved with combination therapy only.

Meaning: Carnosine and hypothermia have synergistic neuroprotective effects against brain damage following $\mathrm{HI}$ injury.

\section{Introduction}

Hypothermia is known to have protective effects against hypoxic-ischemic (HI) brain injury in neonatal animal studies. ${ }^{1-3)}$ Additionally, as a result of large-scale clinical studies of newborns with HI encephalopathy in the early 2000s, therapeutic hypothermia was found to have effects against brain damage caused by perinatal asphyxia. ${ }^{4,5)}$ Subsequently, in 2010, there was an international consensus regarding the application of hypothermia for newborns with HI encephalopathy. ${ }^{6}$ Currently, therapeutic hypothermia has been established as a standard treatment strategy for HI encephalopathy, and it is used for fullterm and some near-term infants with moderate to severe HI encephalopathy. ${ }^{7,8)}$ The neuroprotective actions of hypothermia comprise multifactorial mechanisms, including the reduction of the cell metabolic rate, prevention of high-energy phosphorus compound deficiency, inhibition of excitatory amino acids and inflammatory cytokines, and suppression of programmed cell death and microglial activation at different stages of neuronal cell damage. ${ }^{7,9)}$ Hypothermia is the only treatment method of newborns with $\mathrm{HI}$ brain injury, but the therapeutic benefits are

Corresponding author: Chun Soo Kim, MD, PhD. Department of Pediatrics, Keimyung University School of Medicine, 1035 Dalgubeol-daero, Dalseo-gu, Daegu 42601, Korea

凶Email: cskim@dsmc.or.kr, https://orcid.org/0000-0003-3888-334X

*Current affiliation: Department of Pediatrics, Keimyung University Dongsan Hospital, Daegu, Korea

Received: 4 November, 2020, Revised: 22 January, 2021, Accepted: 5 February, 2021

This is an open-access article distributed under the terms of the Creative Commons Attribution Non-Commercial License (http://creativecommons.org/licenses/bync/4.0/) which permits unrestricted non-commercial use, distribution, and reproduction in any medium, provided the original work is properly cited. 


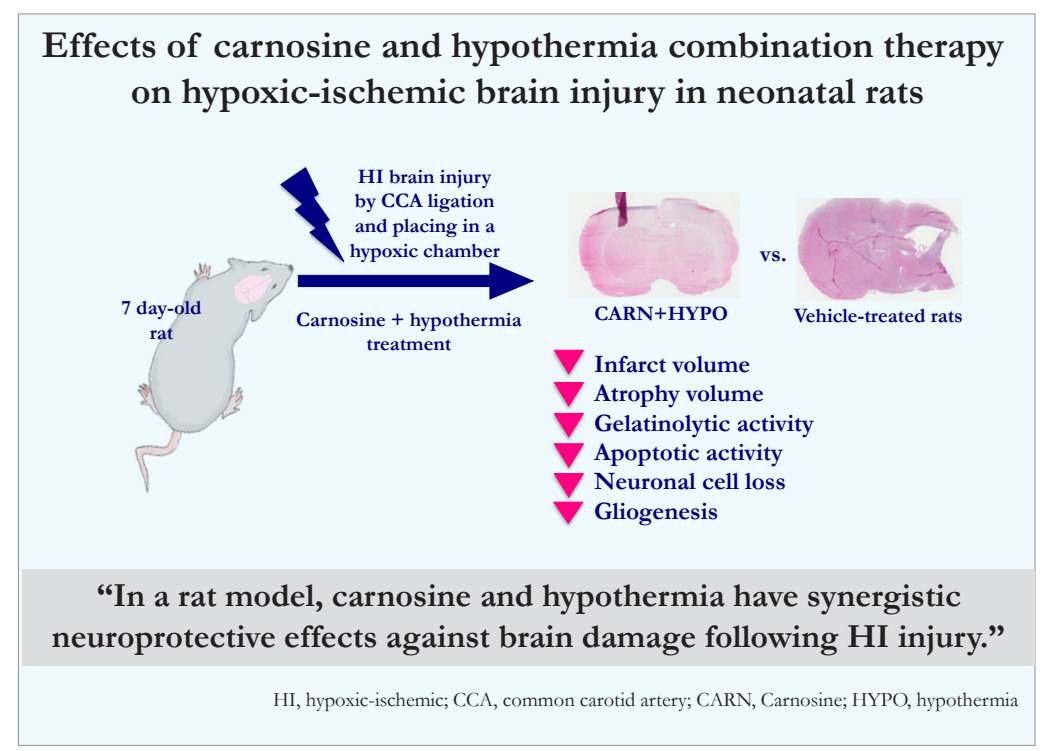

Graphical abstract

still limited. ${ }^{10-12)}$ Recently, combination therapies involving hypothermia and adjunctive agents, including phenobarbital, ${ }^{13)}$ topiramate, ${ }^{14)}$ erythropoietin, ${ }^{15)}$ and xenon, ${ }^{16)}$ have been reported to have better therapeutic effects than hypothermia alone.

Carnosine is an endogenous dipeptide ( $\beta$-alanyl-l-histidine) that is distributed in several parts of the body, including the skeletal and cardiac muscles and nervous tissues. ${ }^{17,18)}$ Recently, carnosine has been shown to have neuroprotective effects against HI brain injury in laboratory and animal studies. In cultured cortical astrocytes under ischemic conditions, carnosine showed the ability to preserving mitochondrial functions by decreasing the intracellular metabolism during the HI phase and improving cell viability by increasing energy production during the reperfusion phase. ${ }^{19)}$ In cultured neurons/astrocytes exposed to oxygen glucose deprivation/recovery, carnosine exhibited neuroprotective effects by regulating extracellular glutamate and gamma-aminobutyric acid levels and mitochondrial energy metabolism. ${ }^{20)}$ During the study of the mouse model, carnosine alleviated brain damage by mechanisms such as inhibiting reactive oxygen species (ROS) and matrix metalloproteinase (MMP) expression of $\mathrm{HI}$ lesions and maintaining glutathione activity. ${ }^{21)}$

Thereafter, this study investigated whether carnosine has additional neuroprotective effects on induced HI brain injury in neonatal rats when used in combination with hypothermic therapy.

\section{Methods}

\section{Experimental protocol}

The study protocol and all experimental procedures were approved by the institutional animal research and ethics committee of Keimyung University School of Medicine (approval number: KM-2014-15). Neonatal Sprague-Dawley rats (KoatechHarlan, Pyeongtaek, Korea) with a body weight of 14 to $18 \mathrm{~g}$ at postnatal day 7 (P7) were used during this study. Anesthesia with isoflurane was administered to induce ischemic brain damage in the rats. After exposing the right common carotid artery of the rat, it was isolated and cut between the double ligations. After surgery, the rats were allowed to recover with the dam for 60 minutes in a cage. Hypoxic injury was caused by placing rats in a chamber at $37^{\circ} \mathrm{C}$ with a flow of mixed gas comprising $8 \%$ humidified oxygen and $92 \%$ nitrogen for 90 minutes under positive pressure.

The study included 2 protocols: protocol I was involved the administration of carnosine or vehicle after $\mathrm{HI}$ injury and hypothermic therapy and protocol II involved the administration of carnosine or vehicle after HI injury without hypothermic therapy (Fig. 1). After inducing brain damage, the rats were transferred to a cage; after 15 minutes, carnosine or vehicle was administered; and rats were allowed to recover with the dam for 60 minutes. Carnosine (Sigma-Aldrich, Seoul, Korea) was dissolved in distilled water and intraperitoneally administered immediately after $\mathrm{HI}$ injury, at a dose of $250 \mathrm{mg}$ / $\mathrm{mL} / \mathrm{kg}$. Additionally, after 24 hours and 48 hours, carnosine or vehicle was administered a total of 3 times. Hypothermia was performed by placing the rats in a chamber maintained at $27^{\circ} \mathrm{C}$ for 3 hours to induce whole-body cooling. The study was divided into the following 4 groups $(n=8-9$ per group): vehicle-treated HI group; carnosine-treated HI group; HI group with vehicle and hypothermia; HI group with carnosine and hypothermia. Sham-treated rats, they were not subjected to any HI injury and included in the study as normal control.

\section{Histological and morphological studies}

Experimental rats were euthanized by decapitation at P10 (3 days after HI injury). Brains were dissected out immediately, and coronal-sectioned specimens were prepared with a thickness of $1 \mathrm{~mm}$ using razor blades. They were then stained with 2\% 2,3,5 triphenyltetrazolium chloride (TTC) solution for 30 minutes at $37^{\circ} \mathrm{C}$, fixed with formalin solution, and photographed with a 
digital camera; the cerebral infarction size was measured using an Image J program (version 1.45; http://rsbweb.nih.gov/ij/ download.html; National Institute Health, Bethesda, MD, USA). The extent of brain infarction was expressed as a percentage of the corresponding area on the undamaged cerebral hemisphere to correct brain edema by the injury.
Experimental rats were also euthanized by decapitation at P35 (4 weeks after HI injury). Brains were dissected out and immediately cooled. Subsequently, $14-\mu \mathrm{m}$-thick coronal slices were prepared using a cryostat (MEV, Mainz, Germany). Sample slides were fixed in formalin for 30 minutes; then, hematoxylin and eosin (H\&E) staining was performed. The brain damage

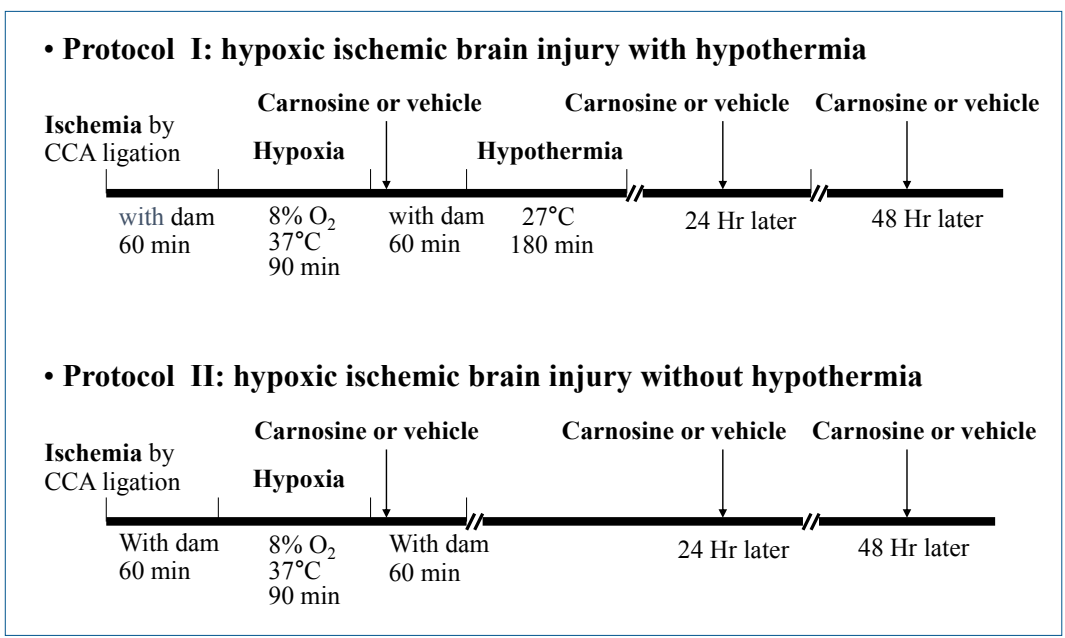

Fig. 1. The diagram shows the experimental processes for protocols I and II. CCA, common carotid artery.
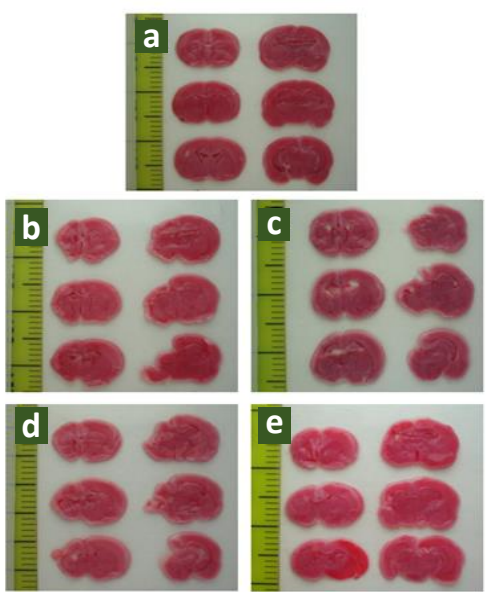

f

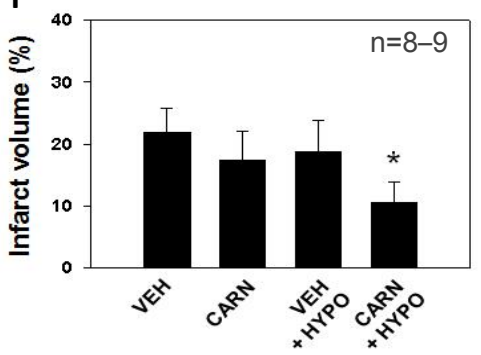

a

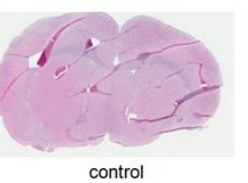

b

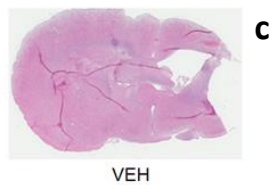

C

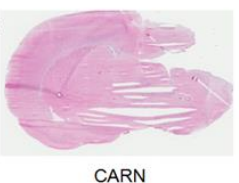

d

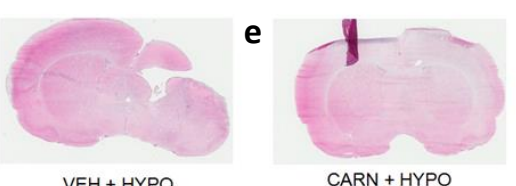

f

B

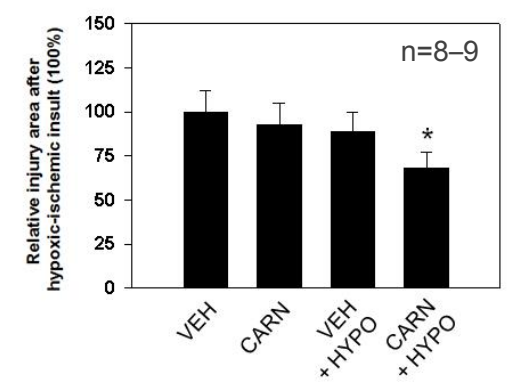

Fig. 2. Histological analysis of hypoxic-ischemic (HI) brain injury in experimental rats. (A) Representative sections of the brain stained with triphenyltetrazolium chloride (TTC) showing infarction areas in P10 rats. Compared to the vehicle (VEH) group, cerebral infarction was significantly reduced with combined carnosine (CARN) and hypothermia (HYPO) therapy. (B) Brain damage findings observed using hematoxylin and eosin staining in P35 rats. Compared to VEH group, brain atrophy was significantly reduced according to the image analysis when a combination of CARN and HYPO was used. P10, postnatal day 10; P35, postnatal day 35; a, sham-treated; b, VEH-treated HI group; c, CARN-treated HI group; d, HI group treated with VEH and HYPO; e, HI group treated with CARN and HYPO. ${ }^{*} P<0.05$ Vs. VEH-treated HI group. 
image was obtained with an optical microscope and digital camera. The brain damage contour was examined using an image analyzer, and the extent of cerebral atrophy was evaluated and compared with the undamaged hemisphere.

\section{In situzymography}

After euthanizing the rats, they were perfused with phosphatebuffered saline (PBS) and brains were rapidly dissected out and frozen in 2-methylbutane and liquid nitrogen. Brain tissue was prepared as section specimens of $14 \mu \mathrm{m}$ using a cryostat, placed in an incubator at $37^{\circ} \mathrm{C}$ for 18 hours according to the instructions of the gelatinase activity assay kit (Enz-Check kit; Invitrogene, Waltham, MA, US), and then examined by fluorescence microscopy.

\section{Terminal deoxynucleotidyl transferase-mediated dUTP nick end-labeling assay}

After euthanizing the rats, they were perfused with PBS and brains were dissected out and frozen in 2-methylbutane and liquid nitrogen. Brain tissue was prepared as section specimens with a thickness of $14 \mu \mathrm{m}$ using a cryostat. Tissue samples were fixed with 10\% formalin solution and washed 3 times with PBS. After following the instructions of the terminal deoxynucleotidyl transferase-mediated dUTP nick end-labeling (TUNEL) staining kit (Roche Diagnostic, Indianapolis, IN, USA) and, adding the reaction solution to the sample tissue, diaminobenzidine substrate solution was added to detect chromogenic apoptosis. Then, the samples were examined using microscopy.

\section{Immunofluorescence}

After euthanizing the rats, they were perfused with PBS, and 4\% paraformaldehyde (PFA) solution were used to fix the brain tissue. Brains were rapidly dissected out and further fixed with 4\% PFA solution; they were soaked with 15\% and 30\% sucrose solution for 24 hours before freezing. Brain tissue was prepared as section specimens with a thickness of $20 \mu \mathrm{m}$ using a cryostat. Slides were prepared and stored in a deep freezer. Samples were blocked with PBS containing 0.3\% Triton X-100 and 3\% normal goat serum. Subsequently, primary antibodies, such as anti-neuronal nuclear antigen (anti-NeuN) (1:100; Millipore, Bedford, MA, USA) or anti-glial fibrillary acidic protein (antiGFAP) (1:200; Millipore), were added to the sample and incubated at $4^{\circ} \mathrm{C}$ for 18 hours. During the next step, a secondary antibody, anti-mouse tetramethylrhodamine-5-isothiocyanate (1:100; Jackson ImmunoResearch, West Grove, PA, USA) was added to the sample and incubated for 30 minutes. Then, the sample was washed with PBS and examined using fluorescence microscopy.
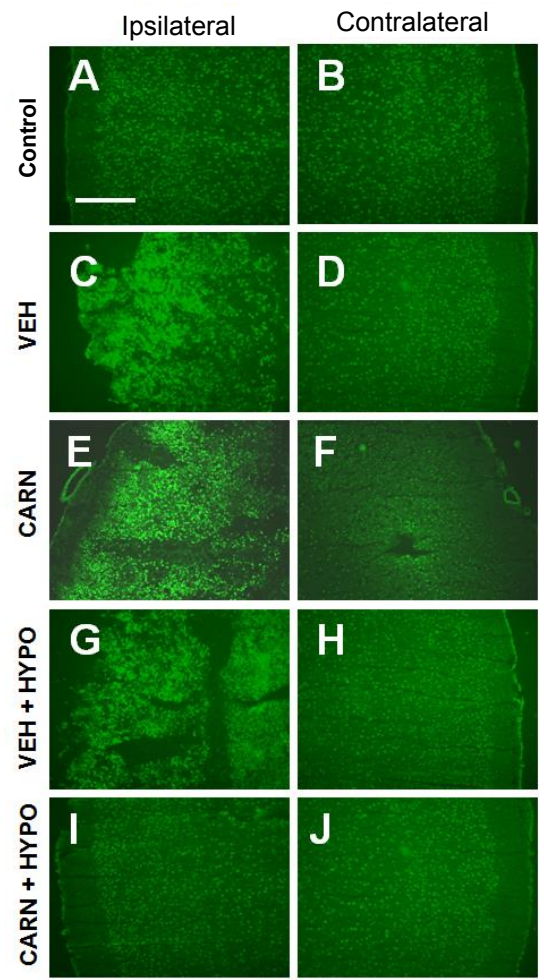

cortex

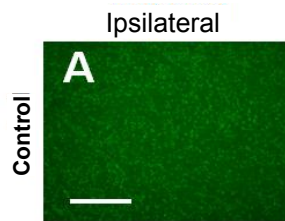

Contralateral
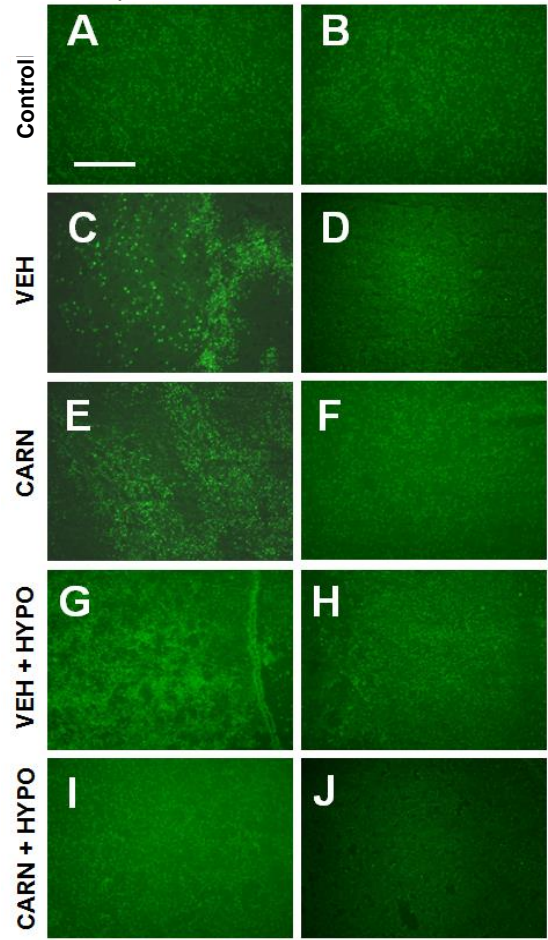

striatum

Fig. 3. Gelatinolytic activity of hypoxic-ischemic $(\mathrm{HI})$ brain lesions detected with in situ zymography in experimental rats. Gelatinase activity of the affected cerebral cortex and corpus striatum increased compared to that of the contralateral sides of the vehicle (VEH) group of P14 rats. Compared to the VEH group, gelatinolysis did not decrease with carnosine (CARN) or hypothermia (HYPO) alone. However, combined CARN and HYPO therapy reduced enzyme activity. P14, postnatal day 14; $A$ and $B$, sham-treated; $C$ and $D, V E H$-treated $H$ group; $E$ and $F, C A R N$-treated HI group; $G$ and $H_{1}, \mathrm{HI}$ group treated with VEH and HYPO; I and J, HI group treated with CARN and HYPO. Scale bar $=500 \mu \mathrm{m}$. 


\section{Statistical analysis}

Data were analyzed using a 1-way analysis of variance and Tukey honestly significant difference test. $P<0.05$ was considered statistically significant.

\section{Results}

\section{Histological and morphological findings}

In the vehicle group of P10 rats, the cerebral infarction area after $\mathrm{HI}$ injury evaluated by TTC staining was $21.9 \% \pm 3.9 \%$. The infarct volume did not decrease significantly with carnosine alone $(17.5 \% \pm 4.6 \%)$ or hypothermia alone $(18.8 \% \pm 5.2 \%)$. Combined carnosine and hypothermia therapy decreased the infarct size $(10.6 \% \pm 3.4 \%)$ compared with vehicle $(P<0.05)$ (Fig. $2 \mathrm{~A})$.

Cerebral atrophy was examined using histological image findings of H\&E stained brain samples of P35 rats. The atrophy area did not decrease significantly with carnosine alone $(91.4 \%$ $\pm 12 \%)$ or hypothermia alone $(87.1 \% \pm 11.5 \%)$. However, com. bined carnosine and hypothermia therapy significantly decreased the lesions of the affected cerebral hemisphere $(67.1 \% \pm 9.7 \%)$ compared with vehicle $(P<0.05)$ (Fig. 2B).

\section{Gelatinolytic activity evaluated by in situzymography}

In the vehicle group of P14 rats, gelatinolytic activity, detected with in situ zymography, of the affected cerebral cortex and corpus striatum increased compared to that of the contralateral sides. Although gelatinolysis did not decrease with carnosine alone or hypothermia alone, combined carnosine and hypothermia therapy reduced the enzyme activity compared with vehicle (Fig. 3).

\section{Apoptosis evaluated by TUNEL assay}

In the vehicle group of P14 rats, TUNEL-positive cells were increased in the affected cerebral cortex compared to the contralateral sides. Although TUNEL-positive cells did not decrease with carnosine alone or hypothermia alone, combined carnosine and hypothermia therapy markedly reduced neuronal apoptosis compared with vehicle. Apoptotic activity was observed even in P35 rats at 4 weeks after injury, and it was also alleviated by combined carnosine and hypothermia therapy (Fig. 4).

\section{Neuroprotective effects evaluated by immunofluorescence staining}

Expression of NeuN-positive cells of the cerebral cortex and corpus striatum in the $\mathrm{P} 35$ vehicle group was decreased compared

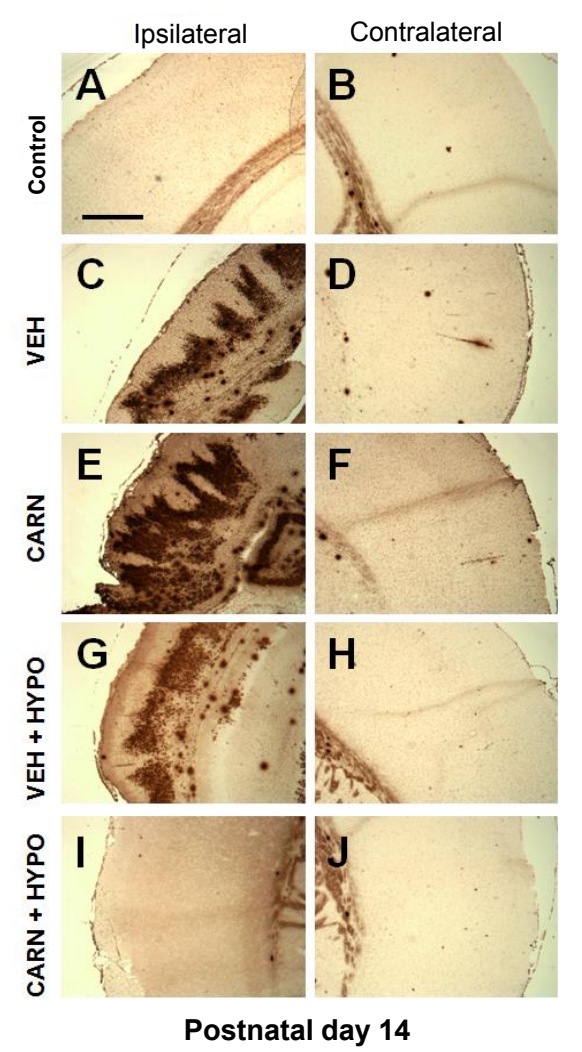

Fig. 4. Apoptosis of hypoxic-ischemic (HI) brain lesions detected by terminal deoxynucleotidyl transferase-mediated dUTP nick end-labeling (TUNEL) staining in experimental rats. TUNEL-positive cells were increased in the affected cerebral cortex compared to the contralateral sides of the vehicle (VEH) group of P14 rats. Compared to the VEH group, TUNEL-positive cells did not decrease with carnosine (CARN) or hypothermia (HYPO) alone. However, combined CARN and HYPO therapy reduced neuronal apoptosis. Apoptotic activity was observed even in P35 rats and was alleviated by combined CARN and HYPO therapy. P10, postnatal day 10; P35, postnatal day 35; A and B, shamtreated group; $C$ and $D, V E H$-treated $H$ I group; $E$ and $F$, CARN-treated $H I$ group; $G$ and $H, H$ group treated with VEH and HYPO; I and J, HI group treated with CARN and HYPO. Scale bar $=500 \mu \mathrm{m}$. 

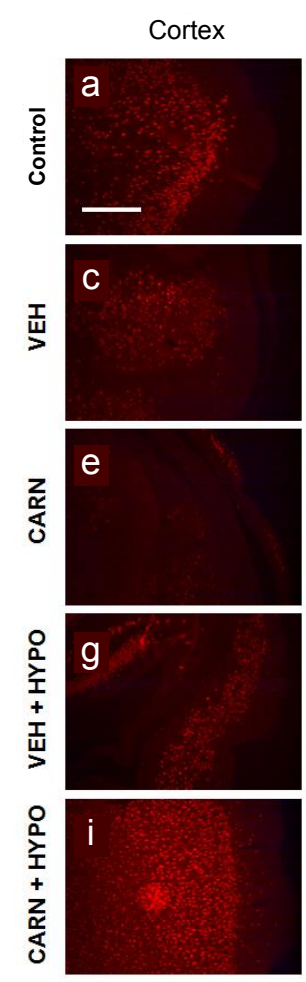

A
Striatum
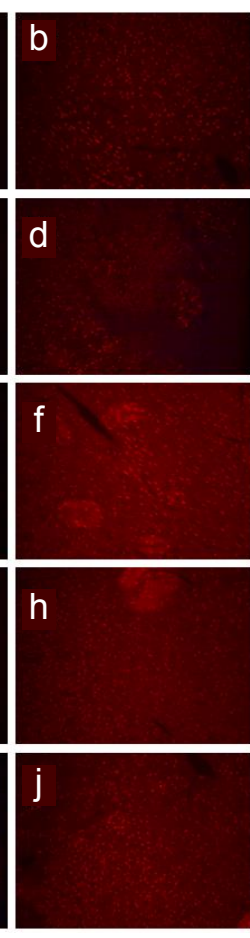

NeuN
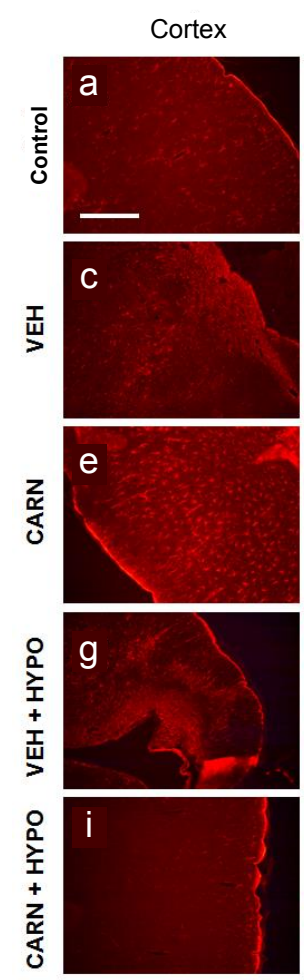

B
Striatum
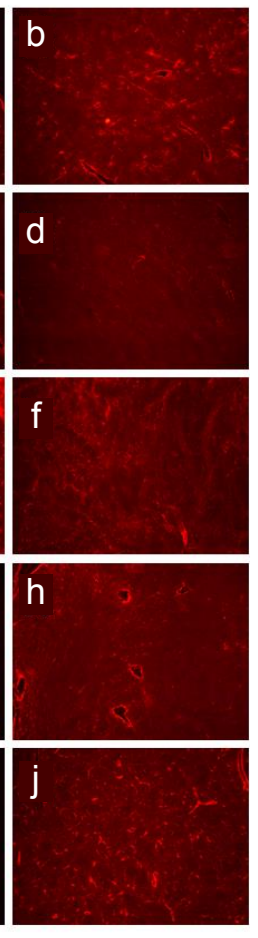

GFAP

Fig. 5. Immunofluorescence findings of hypoxic-ischemic $(\mathrm{HI})$ brain lesions in experimental rats. (A) Expression of neuronal nuclear antigen (NeuN). Neuronal cells of the cerebral cortex and corpus striatum in the P35 vehicle group were decreased compared to sham-treated rats. Compared to vehicle (VEH) group, carnosine (CARN) or hypothermia (HYPO) alone did not prevent NeuN-positive cell loss. However, combined CARN and HYPO therapy reduced neuronal cell loss. (B) Expression of glial fibrillary acidic protein (GFAP). Glial cells of the cerebral cortex and corpus striatum in the P35 VEH group were increased compared to sham-treated rats. Compared to the VEH group, CARN or HYPO alone did not prevent an increase in GFAP-positive cells. However, combined CARN and HYPO therapy suppressed gliogenesis. P35, postnatal day 35; a and b, sham-treated; c and d, VEHtreated HI group; e and f, CARN-treated HI group; $g$ and $h, H$ group treated with VEH and HYPO; i and $\mathrm{j}, \mathrm{HI}$ group treated with CARN and HYPO. Scale bar $=500 \mu \mathrm{m}$.

to sham-treated rats. Carnosine alone or hypothermia alone did not prevent NeuN-positive cell loss. However, combined carnosine and hypothermia therapy reduced neuronal cell loss compared with vehicle (Fig. 5A).

Expression of GFAP-positive cells of the cerebral cortex and corpus striatum in the P35 vehicle group was increased compared to sham-treated rats. Although carnosine alone or hypothermia alone did not prevent an increase in GFAP-positive cells, combined carnosine and hypothermia therapy suppressed gliogenesis compared with vehicle (Fig. 5B).

\section{Discussion}

When comparing the brain development status between rats and humans according to postnatal age, 7-day-old rats are equivalent to term infants, and 10-day-old rats are closely related to infants with $40-42$ weeks gestational age. ${ }^{22,23)}$ Moreover, rats at 15-day-old are equivalent to a few months to 1 year old infants, and rats at 28-33 days-old are correspond to 2-yearold infants. ${ }^{22,24)}$ In this study, combination therapy for P7 rats with $\mathrm{HI}$ brain injury started with the first dose of carnosine administered immediately after the injury. Subsequently, hypothermia was applied for 3 hours; then, secondary and tertiary doses of carnosine were administered. After these interventional treatments, the therapeutic effect against brain damage was evaluated in rats at $\mathrm{P} 10, \mathrm{P} 14$, and $\mathrm{P} 35$ based on the morphological and histological findings of the affected parts. In morphological evaluation, carnosine alone and hypothermia alone did not show any protective effects against $\mathrm{HI}$ brain injury. However, a combination of carnosine and hypothermia morphologically reduced the extent of brain lesions compared with vehicle. Additionally, the results of in situ zymography, TUNEL assays, and immunofluorescence studies showed that neuroprotective effects were achieved with only combined therapy.

According to studies of neonatal rats, neuroprotective effects of hypothermia against $\mathrm{HI}$ brain damage vary depending on the severity of $\mathrm{HI}$ injury, and is also affected by the onset of cooling and the duration of hypothermia. ${ }^{25,26)}$ Sabir et al. ${ }^{12)}$ reported that 5-hour hypothermic therapy for 7-day-old rats with a moderate HI injury is most effective when applied immediately after injury, when applied up to 6 hours after injury, the infarct area increases 
by $1.8 \%$ per hour. However, when hypothermic therapy is applied at 12 hours or more after injury, has no neuroprotective effects. Additionally, for rats with a severe HI injury, hypothermia had no protective effects immediately or within 6 hours after injury. Neuroprotective effects of 3-hour hypothermia after inducing $\mathrm{HI}$ insults in 7-day-old rats differ according to the severity of the injury. Tresche et al. ${ }^{1)}$ reported that there were neuroprotective effects in rats with a mild $\mathrm{HI}$ injury, whereas Liu et al. ${ }^{14)}$ pronounced that there were no therapeutic effects was expressed in rat with moderate $\mathrm{HI}$ injury. In the experimental protocol of this study, neonatal rats were exposed to hypoxia for 90 minutes after ligation of a carotid artery, resulting in moderate $\mathrm{HI}$ brain damage, ${ }^{12)}$ and then the rats were exposed to the environment of $27^{\circ} \mathrm{C}$ to lower the target rectal temperature to $28^{\circ} \mathrm{C},{ }^{14)}$ and the cooling is applied for 3 hours. Therefore, it is considered that neuroprotective effects were not observed with hypothermia alone because cooling was performed for only 3 hours for moderately severe brain injury. In future studies, if a cooling time of 5 hours or more is applied, hypothermia alone is expected to have therapeutic effects. ${ }^{12)}$

During a study of a neonatal rat model by Zhang et al., ${ }^{24)}$ carnosine was administered as a dose of $250 \mathrm{mg} / \mathrm{kg}$ intraperitoneally 3 times immediately after $\mathrm{HI}$ injury, 24 hours later, and 48 hours later. During the evaluation of brain tissues approximately 72 hours after the injury, administration of a series of carnosine doses showed neuroprotective effects by lowering the concentration of prostaglandin F2 $\alpha$ in brain tissue and reducing expression levels of neuronal apoptosis and mitochondrial caspase- 3 protein. In this study, carnosine was administered according to an existing reported protocol, ${ }^{24)}$ but the use of carnosine alone had no neuroprotective effects. Other studies of rodent models have reported that the neuroprotection of carnosine after $\mathrm{HI}$ injury is concentration-dependent and the protective effect is evident when at high doses of 500 to1,000 $\mathrm{mg} / \mathrm{kg}$ were used or when doses were administered prior to brain damage. ${ }^{18,21,27)}$ Therefore, it may be necessary to increase the dosage of carnosine and evaluate the changes in neuroprotective effects bases on the blood concentrations of the therapeutic agent.

To enhance the brain-protective effects of hypothermia after $\mathrm{HI}$ injury, several studies have been conducted to determine whether the administration of combined hypothermia and adjuvant agents has synergistic effects on neuroprotection. Liu et al. ${ }^{14)}$ reported that topiramate administered immediately after $\mathrm{HI}$ injury extends the therapeutic window for hypothermiamediated neuroprotection in a neonatal rat model. In their study that evaluate the brain volume showing cerebral infarction and atrophy after HI injury, topiramate alone and hypothermia alone had no neuroprotective effects; however, a combination of topiramate and hypothermia significantly reduced the extent of cerebral lesions. Martin et al. ${ }^{16)}$ studied a neonatal rat model with $\mathrm{HI}$ injury and reported that asynchronous administration of xenon after hypothermia had a synergistic effect on neuroprotection. In their study, the extent of cerebral infarction after
HI injury did not decrease with hypothermia alone or xenon alone, but the lesion size was significantly reduced when xenon was administered asynchronously at 1- or 5-hour intervals after hypothermia.

In this study, interventional measures performed after the injury had no therapeutic effects with hypothermia alone or adjuvant alone, and protective effects were observed with combined administration, similar to other studies of neonatal rat model of $\mathrm{HI}$ brain injury. ${ }^{14,16)}$ After combined administration of carnosine and hypothermia, gelatinolysis in the brain tissue of the affected side decreased. Neuronal apoptosis was reduced approximately 1 week after the injury, and this reduction was observed even after 4 weeks. Additionally, loss of neuronal cells and gliogenesis of the lesion were suppressed by a combined therapy. This study had some limitations. First, according to the protocol, hypothermia was applied to decrease the target temperature to $28^{\circ} \mathrm{C}$, but the rectal temperature of all rats used in this study was not measured. Furthermore, quantitative analyses of histological changes in brain lesions following therapeutic measures were not performed.

In this study, carnosine was repeatedly administered before and after hypothermia; however, the cellular or molecular mechanisms of carnosine that would increase the neuroprotection of hypothermia is uncertain. Presumably, it is likely related to various biological roles of carnosine against $\mathrm{HI}$ injury, including inhibiting ROS and MMP expression and suppressing microglial activation and neuronal apoptosis. ${ }^{27,28)}$

In conclusion, the combined carnosine and hypothermia therapy in a neonatal rat model of $\mathrm{HI}$ injury synergistically reduced the extent of brain injury. Therefore, it could be a therapeutic strategy for HI encephalopathy of newborns. Further research is needed to elucidate the molecular mechanisms associated with their neuroprotective functions.

\section{Footnotes}

Conflict of interest : No potential conflict of interest relevant to this article was reported.

ORCID :

Jun Chul Byun $(\mathbb{C}$ https://orcid.org/0000-0001-5600-6282

Chun Soo Kim @ https://orcid.org/0000-0003-3888-334X

\section{References}

1. Trescher WH, Ishiwa S, Johnston MV. Brief post-hypoxic-ischemic hypothermia markedly delays neonatal brain injury. Brain Dev 1997;19: 326-38.

2. Roelfsema V, Bennet L, George S, Wu D, Guan J, Veerman M, et al. Window of opportunity of cerebral hypothermia for postischemic white matter injury in the near-term fetal sheep. J Cereb Blood Flow Metab 2004;24:877-86.

3. Xia XY, Xia YX. Effects of graded hypothermia on hypoxic-ischemic brain damage in the neonatal rat. Chin Med Sci J 2011;26:49-53. 
4. Shankaran S, Laptook AR, Ehrenkranz RA, Tyson JE, McDonald SA, Donovan EF, et al. Whole-body hypothermia for neonates with hypoxicischemic encephalopathy. N Engl J Med 2005;353:1574-84.

5. Gluckman PD, Wyatt JS, Azzopardi D, Ballard R, Edwards AD, Ferriero $\mathrm{DM}$, et al. Selective head cooling with mild systemic hypothermia after neonatal encephalopathy: multicentre randomised trial. Lancet 2005;365:663-70.

6. Perlman JM, Wyllie J, Kattwinkel J, Atkins DL, Chameides L, Goldsmith JP, et al. Neonatal resuscitation: 2010 international consensus on cardiopulmonary resuscitation and emergency cardiovascular care science with treatment recommendations. Pediatrics 2010;126:e1319-44.

7. Sung IK. Therapeutic hypothermia for hypoxic-ischemic encephalopathy in newborn infants. Neonatal Med 2017;24:145-56.

8. Chiang MC, Jong YJ, Lin CH. Therapeutic hypothermia for neonates with hypoxic ischemic encephalopathy. Pediatr Neonatol 2017;58:47583.

9. Drury PP, Gunn ER, Bennet L, Gunn AJ. Mechanisms of hypothermic neuroprotection. Clin Perinatol 2014;41:161-75.

10. Silveira RC, Procianoy RS. Hypothermia therapy for newborns with hypoxic ischemic encephalopathy. J Pediatr (Rio J) 2015;91(6 Suppl 1):S78-83.

11. Wyatt JS, Gluckman PD, Liu PY, Azzopardi D, Ballard R, Edwards $\mathrm{AD}$, et al. Determinants of outcomes after head cooling for neonatal encephalopathy. Pediatrics 2007;119:912-21.

12. Sabir H, Scull-Brown E, Liu X, Thoresen M. Immediate hypothermia is not neuroprotective after severe hypoxia-ischemia and is deleterious when delayed by 12 hours in neonatal rats. Stroke 2012;43:3364-70.

13. Barks JD, Liu YQ, Shangguan Y, Silverstein FS. Phenobarbital augments hypothermic neuroprotection. Pediatr Res 2010;67:532-7.

14. Liu YQ, Barks JD, Xu G, Silverstein FS. Topiramate extends the therapeutic window for hypothermia-mediated neuroprotection after stroke in neonatal rats. Stroke 2004;35:1460-5.

15. Fan X, van Bel F, van der Kooij MA, Heijnen CJ, Groenendaal F. Hypothermia and erythropoietin for neuroprotection after neonatal brain damage. Pediatr Res 2013;73:18-23.

16. Martin JL, Ma D, Hossain M, Xu J, Sanders RD, Franks NP, et al. Asynchronous administration of xenon and hypothermia significantly reduces brain infarction in the neonatal rat. Br J Anaesth 2007;98:23640.

17. Boldyrev AA. Carnosine: new concept for the function of an old molecule. Biochemistry (Mosc) 2012;77:313-26.
18. Prokopieva VD, Yarygina EG, Bokhan NA, Ivanova SA. Use of carnosine for oxidative stress reduction in different pathologies. Oxid Med Cell Longev 2016;2016:2939087.

19. Shen Y, Tian Y, Yang J, Shi X, Ouyang L, Gao J, et al. Dual effects of carnosine on energy metabolism of cultured cortical astrocytes under normal and ischemic conditions. Regul Pept 2014;192-193:45-52.

20. Ouyang L, Tian Y, Bao Y, Xu H, Cheng J, Wang B, et al. Carnosine decreased neuronal cell death through targeting glutamate system and astrocyte mitochondrial bioenergetics in cultured neuron/astrocyte exposed to OGD/recovery. Brain Res Bull 2016;124:76-84.

21. Rajanikant GK, Zemke D, Senut MC, Frenkel MB, Chen AF, Gupta R, et al. Carnosine is neuroprotective against permanent focal cerebral ischemia in mice. Stroke 2007;38:3023-31.

22. Jiang W, Duong TM, de Lanerolle NC. The neuropathology of hyperthermic seizures in the rat. Epilepsia 1999;40:5-19.

23. Tucker AM, Aquilina K, Chakkarapani E, Hobbs CE, Thoresen M. Development of amplitude-integrated electroencephalography and interburst interval in the rat. Pediatr Res 2009;65:62-6.

24. Zhang H, Guo S, Zhang L, Jia L, Zhang Z, Duan H, et al. Treatment with carnosine reduces hypoxia-ischemia brain damage in a neonatal rat model. Eur J Pharmacol 2014;727:174-80.

25. Che D, Li L, Kopil CM, Liu Z, Guo W, Neumar RW. Impact of therapeutic hypothermia onset and duration on survival, neurologic function, and neurodegeneration after cardiac arrest. Crit Care Med 2011;39:1423-30.

26. Huh PW, Belayev L, Zhao W, Koch S, Busto R, Ginsberg MD. Comparative neuroprotective efficacy of prolonged moderate intraischemic and postischemic hypothermia in focal cerebral ischemia. J Neurosurg 2000;92:91-9.

27. Xie RX, Li DW, Liu XC, Yang MF, Fang J, Sun BL, et al. Carnosine attenuates brain oxidative stress and apoptosis after intracerebral hemorrhage in rats. Neurochem Res 2017;42:541-51.

28. Ou-Yang L, Liu Y, Wang BY, Cao P, Zhang JJ, Huang YY, et al. Carnosine suppresses oxygen-glucose deprivation/recovery-induced proliferation and migration of reactive astrocytes of rats in vitro. Acta Pharmacol Sin 2018;39:24-34.

How to cite this article: Byun JC, Lee SR, Kim CS. Effects of carnosine and hypothermia combination therapy on hypoxic-ischemic brain injury in neonatal rats. Clin Exp Pediatr 2021;64:422-9. https://doi.org/10.3345/cep.2020.01837 